\title{
Launching a 25-Meter Space Telescope Are Astronauts a Key to the Next Technically Logical Step After NGST?
}

\author{
Mark S. Lake \\ NASA Langley Research Center, M/S 190 \\ Hampton, VA 23681 \\ 310-454-9461 \\ m.s.lake@larc.nasa.gov
}

Abstract The present study considers architectures for a 25 -meter $(25 \mathrm{~m})$ space telescope mirror that leverage the technology development currently underway for the Next Generation Space Telescope (NGST). Key engineering requirements are derived that distinguish a $25 \mathrm{~m}$ mirror from the $8 \mathrm{~m}$ NGST mirror. The feasibility of applying both mirror panel and active-alignment-control technologies currently under development for the NGST to a larger $(25 \mathrm{~m})$ telescope mirror is discussed. Analyses are presented that indicate it is impractical to mechanically deploy a $25 \mathrm{~m}$ telescope mirror simply because of the volumetric packaging inefficiency of such concepts. Instead, it is shown that an erectable mirror, assembled by either astronauts or robotic devices, provides the most logical mirror architecture. Finally, it is shown that an observatory with a $25 \mathrm{~m}$ primary mirror could be launched in one Shuttle mission for assembly and initial checkout in low Earth orbit.

\section{TABLE OF CONTENTS}

1. INTRODUCTION

2. EVOLUTION OF MIRROR TECHNOLOGY

3. DEPLOYMENT VS. ASSEMBLY

4. EVA VS. EVR ASSEMBLY

5. STRUCTURAL REQUIREMENTS

6. SUMMARY

\section{INTRODUCTION}

\section{(WHY CONSIDER A 25M SPACE TELESCOPE?)}

The future science goals of the ultraviolet-optical-infrared (UVOIR) astronomy community include detailed study of the birth and evolution of distant galaxies and planetary systems [1]. The principal space-based observatory under development to make these measurements is the Next Generation Space Telescope (NGST) [2]. With ten times the collecting area of the current Hubble Space Telescope (HST), the NGST will be capable of imaging fainter objects in shorter integration times, and hence will deliver more science through-put than the HST. With a planned $8 \mathrm{~m}$ diameter aperture, the NGST will deliver about the same angular resolution in the near infrared, as the $2.4 \mathrm{~m}$ HST delivers in the visible. Beyond NGST, the UVOIR astronomy community is envisioning even larger filledaperture space telescopes and interferometers. Specifically, 20m-class filled-aperture space telescopes are envisioned after 2010 .

At various times since the early $1960 \mathrm{~s}$, NASA and the astrophysics community have considered the problem of launching 20m-class space telescopes. In the early $1990 \mathrm{~s}$ NASA invested a substantial amount of money in technology development for a $20 \mathrm{~m}$ far infrared space telescope called the Large Deployable Reflector (LDR, see Figure 1) [3]. Like NGST, LDR was to include a segmented primary mirror with an average areal density of about $15 \mathrm{~kg} / \mathrm{m}^{2}$. Recent studies of $20 \mathrm{~m}$-class space telescopes have focused on gossamer telescopes that are extremely lightweight (i.e., areal densities less than 1 $\mathrm{kg} / \mathrm{m}^{2}$ ). Such telescopes would use thin membrane optics coupled with active wave-front control (e.g., Figure 2). Unfortunately, the technology for membrane optics is still very immature, and it is not clear how soon (or even if) such systems will be viable.

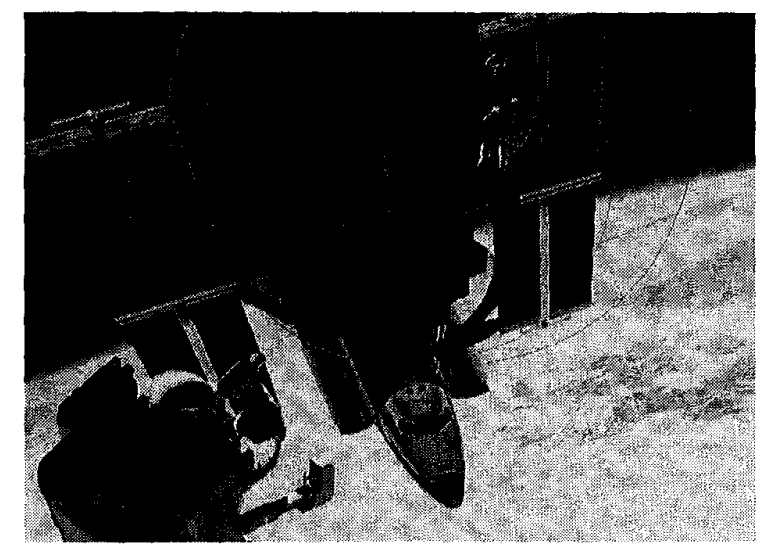

Figure $1-20 \mathrm{~m}$ LDR telescope with segmented mirror

In a sense, the present study is an attempt to define the most technically logical architecture for a $20 \mathrm{~m}$-class space telescope. It is argued that the most technically logical architecture for a $20 \mathrm{~m}$-class space telescope incorporates a segmented primary mirror that takes maximum advantage of the on-going and future technology investments of the NGST program. In addition, the present study is an attempt to illustrate the economies that might be gained by astronaut

\footnotetext{
${ }^{1}$ U.S. Government work not protected by U.S. copyright.

${ }^{2}$ Updated October 15, 2000
} 
involvement in the construction of a $20 \mathrm{~m}$-class space telescope. The motivation behind studying astronaut involvement in telescope construction stems from a desire to capitalize on the extensive on-orbit infrastructure that is soon to be realized at the International Space Station.

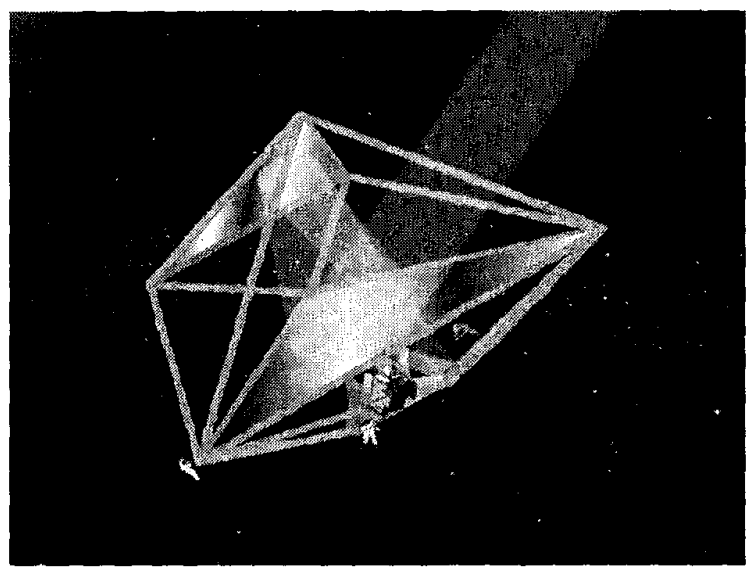

Figure $2-20 \mathrm{~m}$-class gossamer telescope

The present study is focused on the design of a $25 \mathrm{~m}-$ diameter telescope mirror simply because this size gives a tenfold increase in collecting area from the NGST. This study is bounded by the practical goal of applying both mirror panel and active-alignment-control technologies currently under development for the NGST to a $25 \mathrm{~m}$, telescope mirror. Consequently, the present study does not consider gossamer membrane telescope concepts. The present study considers three key system-level issues that will drive the design of a $25 \mathrm{~m}$ space telescope mirror: launch vehicle packaging; on-orbit assembly; and on-orbit structural performance.

\section{EVOLUTION OF MiRRoR TECHNOLOGY (The Technology Push of NGST)}

Until membrane optics become viable for large space telescopes, it is logical to continue the development of large telescope concepts that use lightweight, segmented optics. Current mirror-development programs for NGST and the Far Infrared Space Telescope (FIRST) are expected to result in space-qualified, segmented optics that are an order of magnitude lighter than the HST primary mirror. In fact, the primary mirrors for these next-generation instruments will be so light that they will occupy more volume in the launch vehicle payload bay than they occupy mass (i.e., they will be volume-critical as opposed to mass-critical).

\section{Reduction of Areal Density to enable the $8 m$ NGST Mirror}

A comparison of the specifications for the $8 \mathrm{~m}$ NGST primary mirror with those for the $2.4 \mathrm{~m}$ HST primary mirror demonstrates the dramatic advancement that is anticipated in telescope mirror technology as a consequence of the NGST program. At roughly $750 \mathrm{~kg}$ each, the primary mirrors for both HST and NGST have about the same total mass despite the tenfold difference in collecting area [2]. However, total mass is about the only similarity between the two mirrors.

A key difference between the HST and NGST primary mirrors is that the HST mirror used an almost insignificant portion of its launch vehicle capacity, whereas the NGST mirror is expected to be the dominant component within its launch package. This difference in launch vehicle utilization is the primary driving force behind efforts within the NGST program to achieve an areal density of approximately 15 $\mathrm{kg} / \mathrm{m}^{2}$, as compared to the $175 \mathrm{~kg} / \mathrm{m}^{2}$ areal density of HST.

The HST mirror occupied only about $4 \%$ of the launch mass capacity of its launch vehicle (i.e., the Space Shuttle), whereas the NGST mirror is expected to occupy on the order of $15 \%$ of the launch mass capacity of its launch vehicle (e.g., the Atlas IIARS). Likewise, the HST primary mirror occupied less than $2 \%$ of the payload bay volume of the Space Shuttle whereas the NGST is expected to occupy over $50 \%$ of the payload bay volume of its launch vehicle!!

\section{Improvement in Packing Efficiency to Enable $25 \mathrm{~m}$ Mirrors}

For a $25 \mathrm{~m}$-telescope mirror to be efficiently launched, both packaged volume and mass must be minimized. Furthermore, it is important to balance the allocation of mass and volume in order to make efficient use of the launch vehicle. One way of illustrating this point is depicted in Figure 3. Lines of constant slope in Figure 3 represent different values of the average payload density (i.e., the total mass of the payload divided by the total volume it occupies in the launch shroud). A cursory review of the launch vehicle literature indicates that the average payload density delivered to low Earth orbit (LEO) by most medium to heavy launch vehicles is about $65 \mathrm{~kg} / \mathrm{m}^{3}$ [6]. For example, both the Space Shuttle and the Atlas IIARS deliver approximately $65 \mathrm{~kg} / \mathrm{m}^{3}$ to Low Earth Orbit. This average payload capacity is indicated by the bold line in Figure 3, and the two lines at 20 and $200 \mathrm{~kg} / \mathrm{m}^{3}$ represent a one-orderof-magnitude variance band around $65 \mathrm{~kg} / \mathrm{m}^{3}$.

Figure 3 compares the masses and packaged volumes of HST, NGST, and two $25 \mathrm{~m}$ radio frequency (RF) reflector designs (TRW s AstroMESH“ mesh reflector [4], and JPL $s$ ARISE inflatable reflector concept [5]). The horizontal axis in Figure 3 represents the areal density of the reflectors, and the vertical axis represents the volumetric package efficiency of the reflectors (quantified as the average spacing between layers of the reflector in the launch package). The package densities of the two $25 \mathrm{~m} \mathrm{RF}$ reflectors included in Figure 3 are about 40 and $50 \mathrm{~kg} / \mathrm{m}^{3}$, respectively, which are fairly close to the average launch vehicle capability of $65 \mathrm{~kg} / \mathrm{m}^{3}$. These data indicate that a launch-package density goal of $65 \mathrm{~kg} / \mathrm{m}^{3}$ is reasonable for large apertures. 


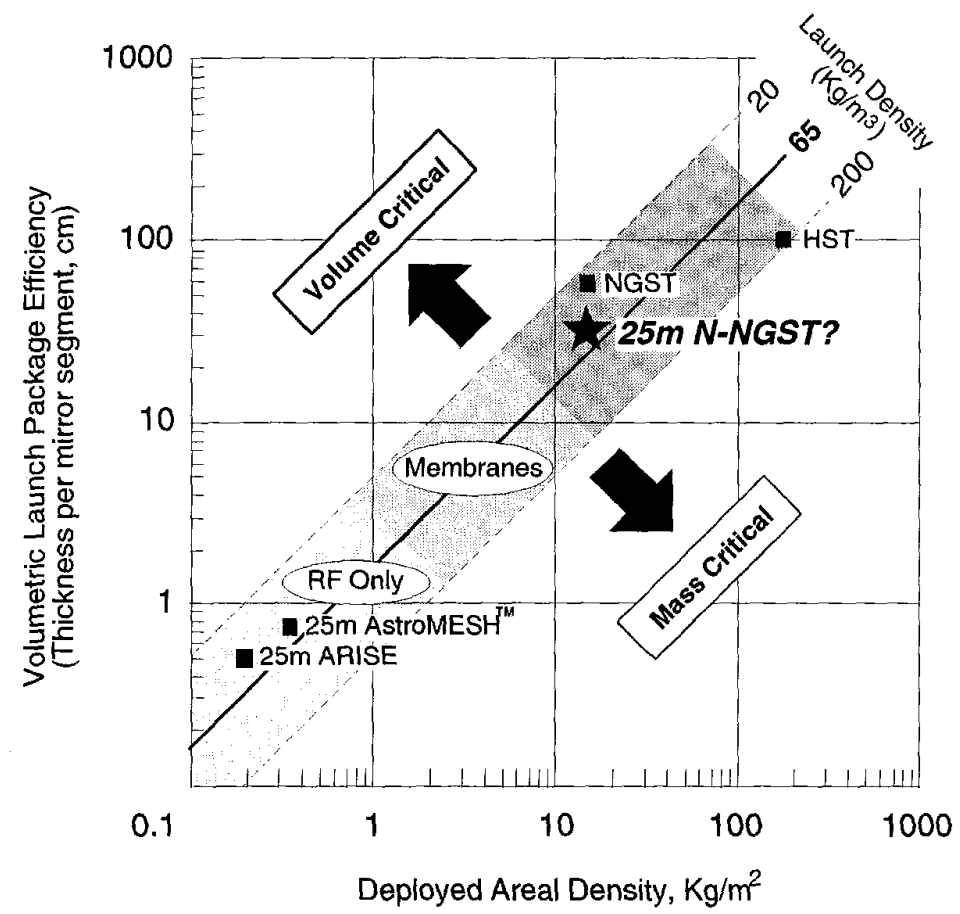

Figure $3-$ Evolution of telescope mirrors quantified by launch mass and volume

The data points in Figure 3 labeled HST and NGST indicate the evolution in technology from HST to NGST. As mentioned, NGST represents over an order of magnitude reduction in areal density, and through its use of mechanical deployment, NGST is packaged more efficiently than the monolythic HST mirror. The NGST primary mirror will have an average density in the launch package of only about $25 \mathrm{~kg} / \mathrm{m}^{3}$ whereas the HST mirror had a launch density of almost $200 \mathrm{~kg} / \mathrm{m}^{3}$. Hence, the HST mirror was mass critical, whereas the NGST mirror will be volume critical.

Figure 3 also includes a star denoting the approximate areal density and packaging efficiency that will be shown herein to be adequate for a $25 \mathrm{~m}$ successor to NGST (N-NGST). In the next section, it will be shown that a $25 \mathrm{~m}$ mirror will require the development of more efficient packaging schemes than the simple deployment concepts currently envisioned for NGST. Specifically, it will be shown that a $25 \mathrm{~m}$ mirror requires the use of an erectable rather than a deployable architecture.

\section{DEPLOYMENT VS. ASSEMBLY (The Problem of PACKaging a 25M Mirror)}

Segmented reflectors must be mechanically deployed or erected on-orbit by astronauts or robotic devices. Mechanical deployment is acceptable for NGST because an $8 \mathrm{~m}$ mirror can be packaged in a $3 \mathrm{~m}$ to $4 \mathrm{~m}$ launch shroud using simple deployment kinematics, and a minimal number of deployment mechanisms. However, it is much more difficult to design a $25 \mathrm{~m}$ mirror that can deploy out of a $3 \mathrm{~m}$ to $4 \mathrm{~m}$ launch shroud.
Illustrative Example of a Large Deployable Reflector

In the past, numerous concepts for mechanical deployment of $20 \mathrm{~m}$-class mirrors have been studied. One example that illustrates the packaging problem of large deployable mirrors is the Starburst mirror discussed in [7] and shown in Figure 4. The Starburst mirror includes 36 panels supported on six booms connected to a central hub. Each of the six booms support six panels and provides the hinge and latch hardware for deployment and latching of the panels. Like all large deployable mirrors, the Starburst design includes significant space between adjacent mirror panels in the launch package to preclude contact between the panels during deployment. This empty space leads to large package volumes.

The launch package and deployed geometry of the Starburst reflector are defined in Figure 5. From these dimensions, the volume of the launch package is:

$$
\text { Volume }=\pi \frac{81}{32} L^{3}=\pi \frac{81}{32}\left(\frac{D}{7}\right)^{3}
$$

Assuming the mass of the mirror is given by the total collecting area of the mirror times its average areal density, the mass of the Starburst reflector is:

$$
\text { Mass }=\rho_{\text {areal }} \frac{3 \sqrt{3}}{2}\left(\frac{\mathrm{D}}{2}\right)^{2}
$$



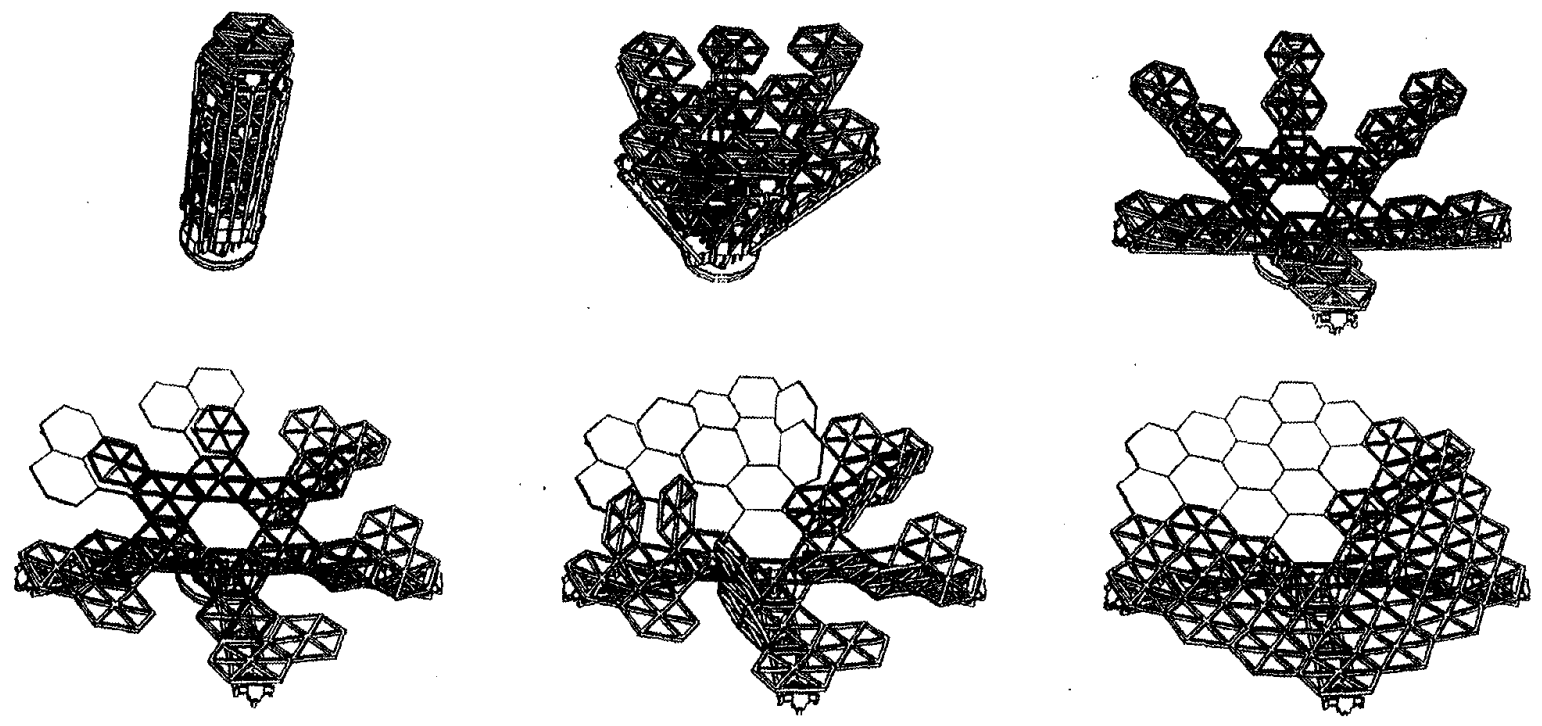

Figure 4 - "Starburst" deployable reflector concept [7]

Note that the volume of the Starburst launch package increases with the cube of the diameter whereas the mass increases with only the square of the diameter. This leads to decreasing package density as the deployed diameter increases. This result follows from the assumption that the deployed areal density is independent of diameter.
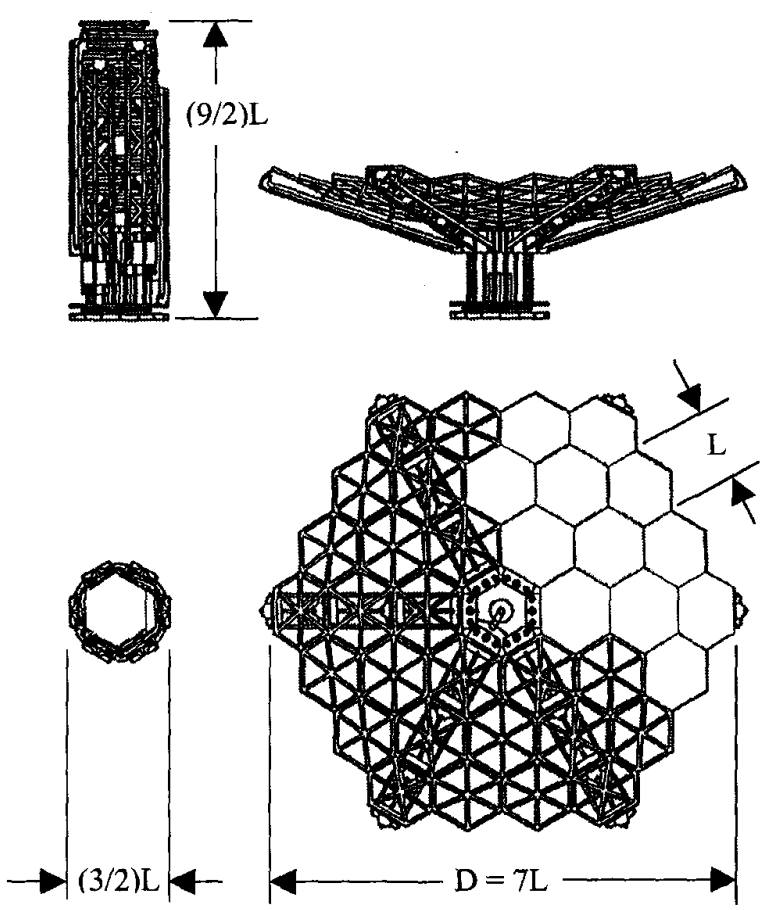

Figure 5 - Package volume dimensions for Starburst [7]

Figure 6 is a plot of the launch package volume and mass determined from Equations (1) and (2), assuming an areal density equal to that expected for the NGST ( $\rho_{\text {areal }}=15$ $\mathrm{kg} / \mathrm{m}^{2}$ ). For simplicity, the launch volume is normalized by the volume of the Shuttle cargo bay $\left(310 \mathrm{~m}^{3}\right)$, and the launch mass is normalized by the available payload mass of the Shuttle $(20,000 \mathrm{~kg})$. Figure 6 illustrates the volumetric inefficiency of the Starburst launch package for mirrors larger than $10 \mathrm{~m}$. In fact, a $21 \mathrm{~m}$ diameter Starburst exhibits a package density of only $20 \mathrm{~kg} / \mathrm{m}^{3}$ - well below the optimum density of $65 \mathrm{~kg} / \mathrm{m}^{3}$ defined in Figure 3 .

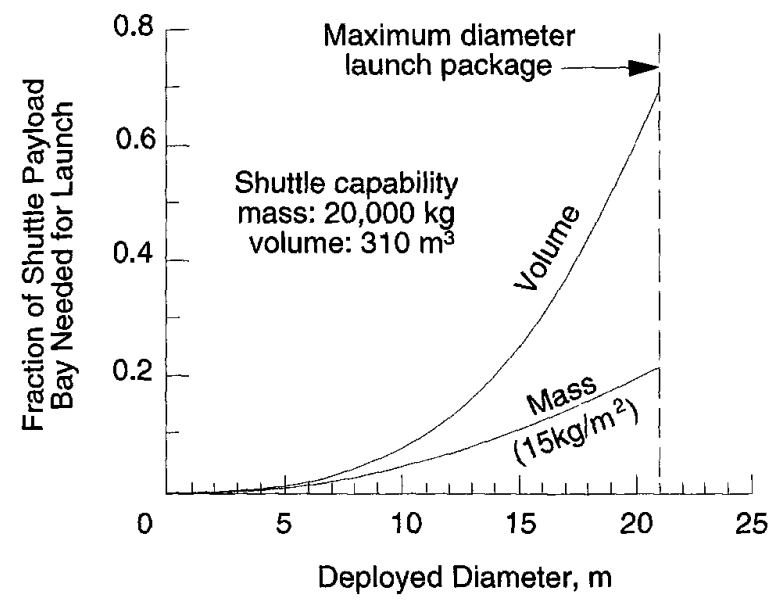

Figure 6 - Launch mass and volume estimates for a Starburst deployable reflector with $\rho$ areal $=15 \mathrm{~kg} / \mathrm{m}^{2}$

From Figure 5, the ratio of the deployed diameter to the stowed package diameter for the Starburst design is approximately $4^{2} / 3$ to 1 . This result means that the largest Starburst reflector that could be packaged in the $4.6 \mathrm{~m}$ diameter Shuttle (or Titan IV) cargo bay is about $21 \mathrm{~m}$ in diameter. A $25 \mathrm{~m}$-diameter version of the Starburst would require a slightly more efficient packaging geometry. 
Nevertheless, the package volume calculations for the Starburst design are adequate for comparing deployment and assembly of a $20 \mathrm{~m}$-class reflector.

\section{Illustrative Example of a Large Erectable Reflector}

It has been recognized that the poor packaging efficiency of mechanical deployment systems is one of their key disadvantages for application to large segmented mirrors. In 1990 Mikulas [8] concluded that:

The ability to tightly package the panels is a major benefit of the erectable approach to constructing large segmented mirrors.

The erectable reflector concept discussed in [8] is sketched in Figure 7, and includes the same arrangement of 36 hexagonal reflector panels included in the Starburst concept. Instead of being supported on a mechanically deployable back structure, the erectable reflector includes a doubly curved tetrahedral truss structure comprised of 96 joints and 315 struts. Each strut in the truss has approximately the same length as the flat-to-flat dimension of one of the panels (i.e., L in Figure 7).

\section{Launch Packages}

Panel package
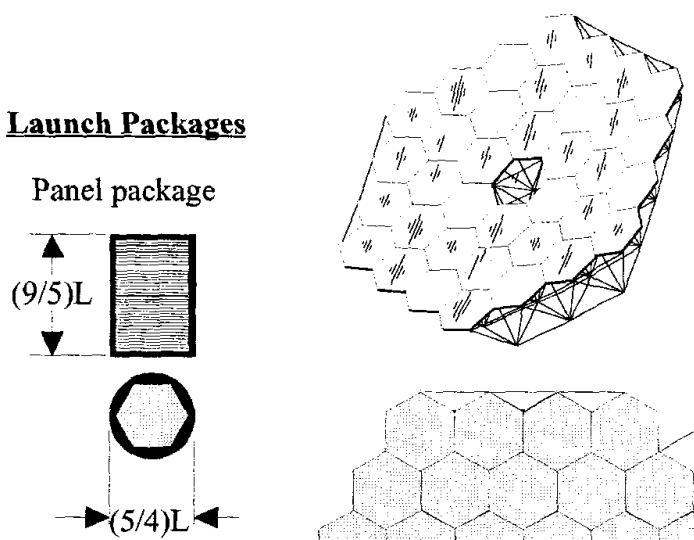

Truss package
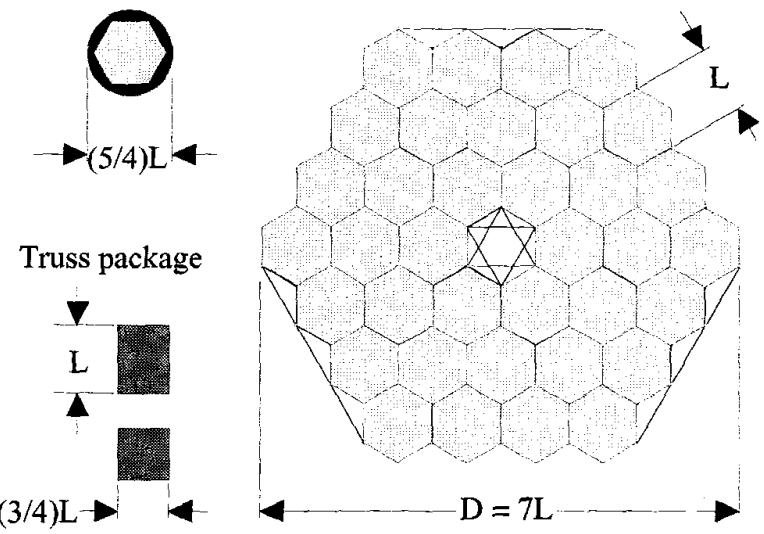

Figure 7 - Approximate dimensions for erectable reflector

The key to the packaging efficiency of an erectable reflector is that its parts can be packaged with very little empty space between them. Hardware studies in [8] assume that the erectable truss struts should have a length-to-diameter ratio of no less than 1/40, and could easily be packaged for launch in roughly four times their volume (i.e., twice their cross-sectional diameter). Similarly, the reflector panels should have a thickness-to-diameter ratio of $1 / 40$, and be packaged in twice their thickness. These rules-of-thumb are reflected in the launch package volumes shown graphically in Figure 7 and used in the following equation for total launch package volume:

$$
\text { Volume }=\frac{45 \pi+36}{64}\left(\frac{D}{7}\right)^{3}
$$

Similar to the case for the Starburst deployable reflector, the packaged volume of the erectable reflector increases with the cube of the diameter. For simplicity, it can be assumed that the mass of the erectable reflector is given by Equation (2). In other words, the mass of either erectable or deployable reflectors are calculated in the same way, and is equal to the total collecting area of the reflector times the average areal density (which is assumed to be $15 \mathrm{~kg} / \mathrm{m}^{2}$ in this case).

Figure 8 presents a plot of the normalized launch mass and volume of the erectable reflector [given by Equations (2) and (3)]. The launch volume advantage expected from an erectable reflector can be clearly seen by comparing the package volume curves in Figures 6 and 8 . The erectable reflector packs into roughly $1 / 3$ of the volume as the Starburst deployable reflector. Although this difference is not of great consequence for mirrors up to about $10 \mathrm{~m}$ in diameter, the difference is dramatic for mirrors larger than $10 \mathrm{~m}$ in diameter.

For the numbers assumed herein, the normalized volume and mass of the erectable reflector launch package are equal for a mirror of about $19 \mathrm{~m}$ in diameter. In other words, a $19 \mathrm{~m}$ erectable reflector can be packaged in the optimum packaging density of about $65 \mathrm{~kg} / \mathrm{m}^{3}$. These analyses indicate that a $25 \mathrm{~m}$ diameter erectable reflector could be designed to package in an average density of about $50 \mathrm{~kg} / \mathrm{m}^{3}$ - occupying about 40 percent of the Shuttle payload volume capacity and 30 percent of the payload mass capacity.

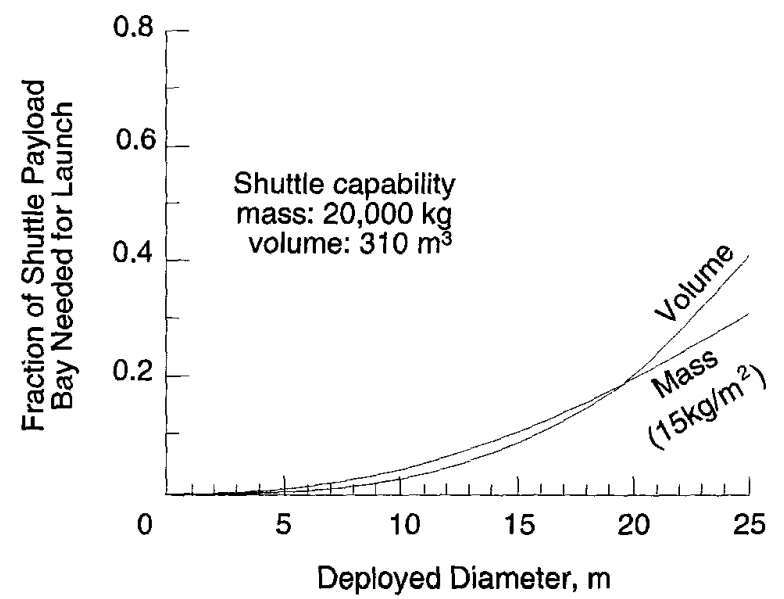

Figure 8-Launch mass and volume estimates for an erectable reflector with $\rho_{\text {areal }}=15 \mathrm{~kg} / \mathrm{m}^{2}$ 
The basic result illustrated by these package volume calculations is that small to moderate diameter reflectors (e.g., up to $10 \mathrm{~m}$ diameter) can be packaged with adequate efficiency using mechanical deployment schemes. However, large diameter mirrors (e.g., above $10 \mathrm{~m}$ ) require erectable assembly architectures to achieve an adequate packaging efficiency in the launch vehicle. A $25 \mathrm{~m}$ erectable reflector could be packaged in less than half of one Shuttle payload bay whereas a $25 \mathrm{~m}$ version of the Starburst deployable reflector will not fit in the Shuttle payload bay.

\section{EVA VS. EVR ASSEMBLY (A QUESTION OF AVAILABILITY AND CAPABILITY)}

It has been demonstrated that, in order to achieve a reasonably efficient launch package, it is more feasible to erect on-orbit (rather than mechanically deploy) a $25 \mathrm{~m}$ diameter segmented telescope mirror. Methods for erecting a $25 \mathrm{~m}$-telescope mirror on-orbit will now be considered.

Conceptually, either extravehicular robotic (EVR) devices or astronauts in extravehicular activity (EVA) could assemble a $25 \mathrm{~m}$-telescope mirror. Although there are many issues that define the economics and capability of EVA and EVR systems, common wisdom holds that EVR systems are best suited for jobs involving a large number of tasks whereas EVA is best suited for a small to moderate number of tasks. With this in mind, one can consider the problem of on-orbit assembly of a telescope mirror from the perspective of the number of parts to be assembled, and hence the number of assembly tasks.

From this perspective, small erectable reflectors (i.e., less than $10 \mathrm{~m}$ in diameter) with a small number of parts (i.e., tens of parts) could easily be constructed by EVA astronauts. Very large reflectors (i.e., larger than $100 \mathrm{~m}$ in diameter), with thousands of parts would require EVR assembly. Moderately large reflectors (i.e., $10 \mathrm{~m}$ to $50 \mathrm{~m}$ ) with hundreds of parts could conceivably be assembled using either EVA or EVR systems. For mirrors of this size, selection between EVA and EVR assembly should be based on both practical and economic considerations. These considerations include issues such as technology readiness, development costs, and the availability of EVA versus EVR systems for a particular mission application (e.g., if the telescope is to be assembled in an orbit inaccessible by astronauts, robots are the only viable option).

During the early $1990 \mathrm{~s}$ NASA Langley Research Center studied both the EVR and EVA assembly methods, and the pros and cons of both methods were derived from extensive laboratory tests of prototype systems. Some results from these research programs are discussed here to illuminate key considerations in the development of tools and procedures for either assembly method.

\section{EVA Assembly of a Large Erectable Reflector}

In 1992, a 14m, 36-panel segmented reflector was assembled in simulated EVA (i.e., neutral buoyancy) in order to study specifically the problems associated with EVA assembly of such a structure (Figure 9). The mirror test article was similar to the erectable mirror described in Figure 7 (e.g., it included a doubly curved support truss with 315 members). The complete results of this test are presented in [10], and are applicable to the assembly of a $25 \mathrm{~m}$ reflector.

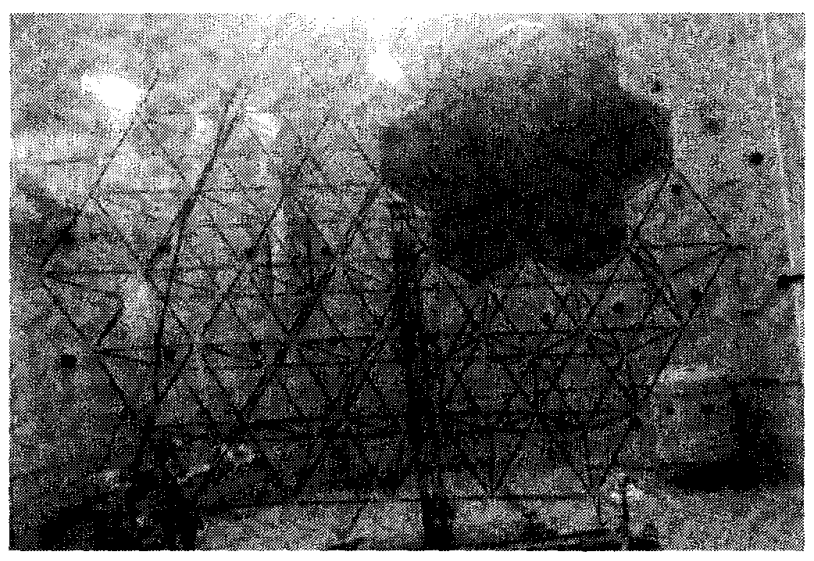

Figure $9-14 \mathrm{~m}$ erectable reflector assembled in simulated EVA test [10]

The EVA assembly test program was designed to address specific concerns or risks associated with EVA assembly of a large precision reflector. Among these concerns, the top two are: 1) the risk of damage to the mirror panels during assembly; and 2) the large amount of EVA time potentially needed to accomplish the assembly. These concerns were mitigated through the development of careful packaging and handling procedures, and efficient assembly procedures. Several additional concerns, such as the risk of interchanging parts and the time needed to position the astronauts at a large number of work sites, were also addressed in this test program.

The overall conclusion of the 1992 test program was that EVA assembly of a large precision reflector is a challenging task, but certainly within the capability of current EVA technology. Specifically, engineering test subjects were able to assemble the $14 \mathrm{~m}$-diameter reflector (Figure 9) in approximately 3 hours of simulated EVA time, and the only infrastructure necessary to support the EVA assembly was a pair of moving foot restraints and positioning devices for hardware canisters. Although these devices don t currently exist for either the Shuttle or the Space Station, they could be developed at relatively low cost.

\section{EVR Assembly of a Large Erectable Reflector}

Also during the early $1990 \mathrm{~s}$, an $8 \mathrm{~m}$-diameter segmented reflector test article made of similar-sized (i.e., $2 \mathrm{~m}$ ) truss struts and reflector panels was assembled in a one-g laboratory environment using a prototype of an EVR system (Figure 10). Just as in the case of the simulated EVA assembly tests, the simulated EVR tests were conducted to 
study the problems associated with assembly of a precision reflector using an EVR system.

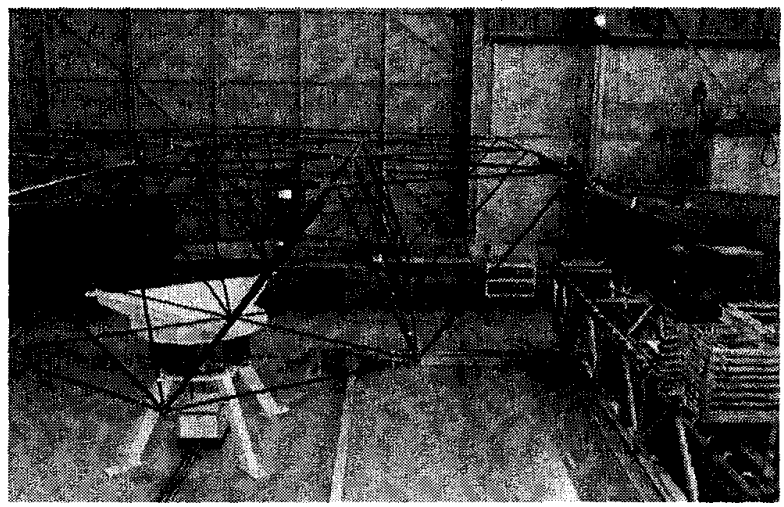

Figure $10-8 \mathrm{~m}$ erectable reflector assembled in simulated EVR test [11]

The EVR assembly test program was designed to address specific concerns or risks associated with EVR assembly of a large precision reflector. The primary concerns relate to the reliability and efficiency of the automated system. Specifically, the mechanical assembly operations and the interfaces between the robotic system and the reflector hardware had to be designed to be robust, and a detailed fault detection and correction system of protocols had to be developed to ensure overall reliability. Several additional concerns, such as the efficient interaction by a remote operator and the time needed to position the robotic devices at a large number of work sites, were also addressed in this test program.

The overall conclusion of the test program was that EVR assembly of a large precision reflector is a challenging task, but likely to be within the capability of future EVR technology. The reason that EVR systems are viewed as less ready than EVA is simply due to the fact that we currently have little or no flight-qualified EVR infrastructure. Specifically, development of an EVR assembly system would require development of moving platforms and work stations (just as required for EVA assembly), PLUS development of the robotic manipulators, software control systems, etc.

\section{General Comparison between EVR and EVA Assembly}

In both EVA and EVR test programs, very detailed assembly procedures were developed and validated, timelines for assembly rates were derived, and performance of flight-like reflector hardware was evaluated. The test programs showed that both EVR and EVA assembly place similar demands on the design of the reflector hardware. One of the more challenging pieces of reflector hardware to design is the latch that is used to attach the reflector panels to the truss. Prototype panel latch designs were evaluated during these test programs, and salient operational features were identified. Figure 11 shows a picture of the EVA panel attachment latch that was evaluated in the EVA assembly test program.

This latch is critical because it must provide a kinematic interface between the panel and the truss, and it must allow for reasonable misalignment during assembly while providing for easy EVA/EVR operation. These requirements are similar to the requirements that have been placed on several of the EVA latches used during servicing of the HST. Hence, there is no reason to question whether a flightqualified version of such a latch could be developed.

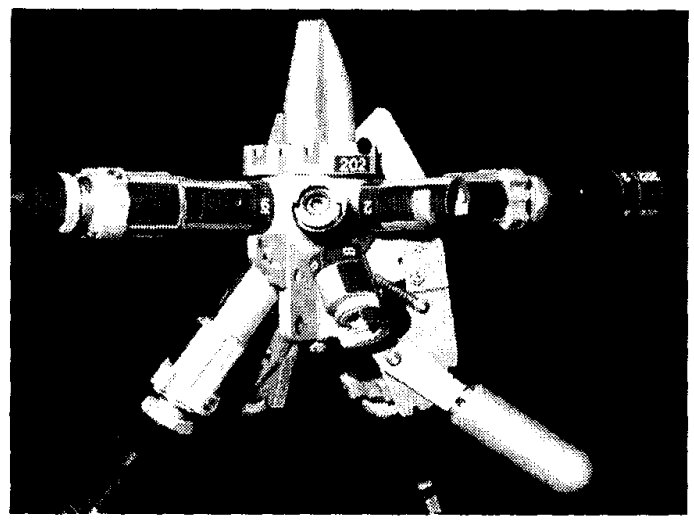

Figure 11 - EVA panel attachment latch [10]

Another similarity between EVR and EVA assembly systems is that they both require attention to the risk of damaging reflector panels. In particular, both assembly approaches require the development of storage and handling procedures for the panels that preclude risk of damage. For example, the panels were only handled from their back side and only attached to the structure after a sufficient number of truss members had been installed to provide three hardpoints to accept the panel (see Figure 12). The key piece of hardware in the development of these procedures is the panel-attachment latch discussed previously.

One final similarity between EVA and EVR assembly systems is the need for ease of operation of the reflector hardware. Data from both test programs show that over half of the total time necessary for assembly of the reflector is consumed while making the mechanical connections between truss components or between the reflector panels and the truss. (The remainder of the time includes all other operations such as repositioning of the work site, and dispensing of the hardware from the storage canisters.)

Figure 13 includes photographs, which illustrate the main drivers of EVA and EVR truss-assembly times. For EVA assembly, the restriction of the pressure suit and the difficulty in getting access to some structural connectors are the main drivers of the assembly rate (and astronaut fatigue). For EVR assembly, the path management and collision-avoidance systems necessary to ensure safe assembly are the main drivers of assembly rates. 


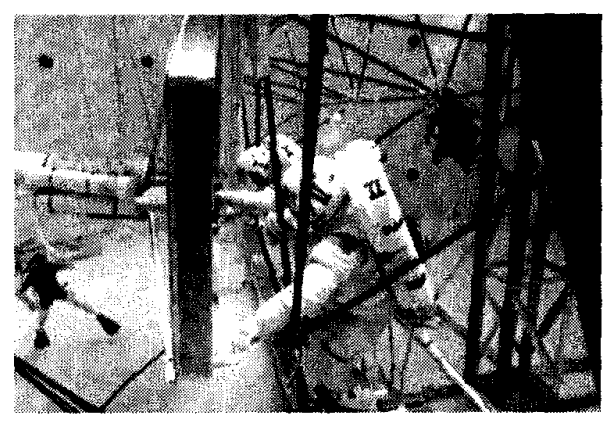

(a) - EVA procedures

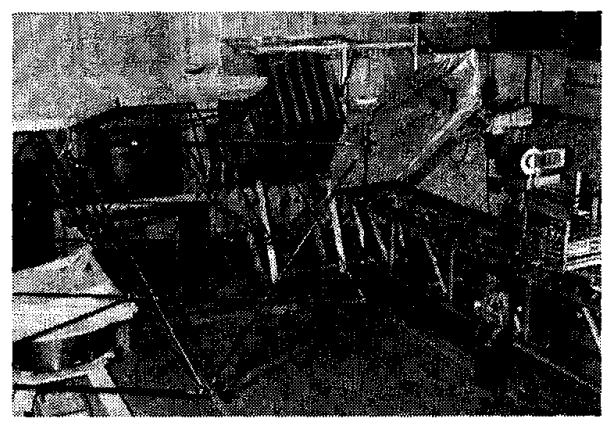

(b) - EVR procedures

Figure 12 - Key procedure for both EVA and EVR assembly is precluding damage during panel installation

In both EVA and EVR assembly, it was found that once the structural component (e.g., truss strut) had been maneuvered into alignment for assembly, significant time was often required to effect final lockup of the joint hardware. As a consequence, it was determined that the operability of the mechanical joints to be assembled in EVA or EVR is a very important aspect of their performance that should be evaluated during development. For EVA assembly, efficiency and speed of assembly is critical - especially as the part count and the number of mechanical joints increases with aperture diameter. For EVR assembly, speed of assembly is not AS critical, but is still a concern.

Figure 14 presents estimates for the total time necessary to build a $25 \mathrm{~m}, 36$-panel precision reflector with EVA and EVR systems. The estimates are based on the test results published in [10] and [11], and include multiplication factors to compensate for the size of the reflectors built in these tests. These estimates show roughly a factor of five difference between the total assembly time for EVA and EVR systems. This difference is attributable to the slower pace of operations of an EVR system necessary to ensure reliable and accurate execution of the tasks. However, as mentioned previously, the difference in assembly times should not be viewed as a big discriminator between assembly systems. Longer assembly times are tolerable in an EVR system because astronaut fatigue and risk are not a concern.

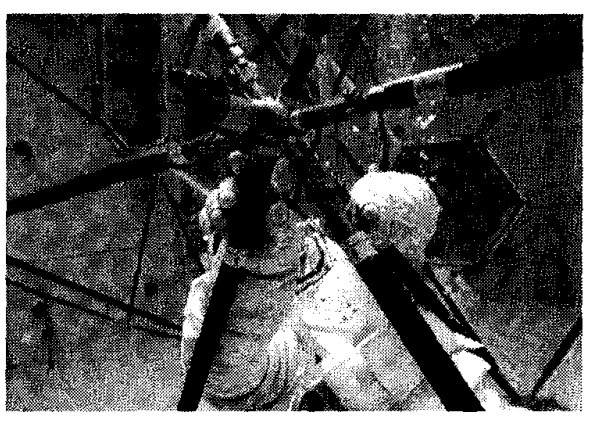

(a) - Difficulteach during EVA truss assembly

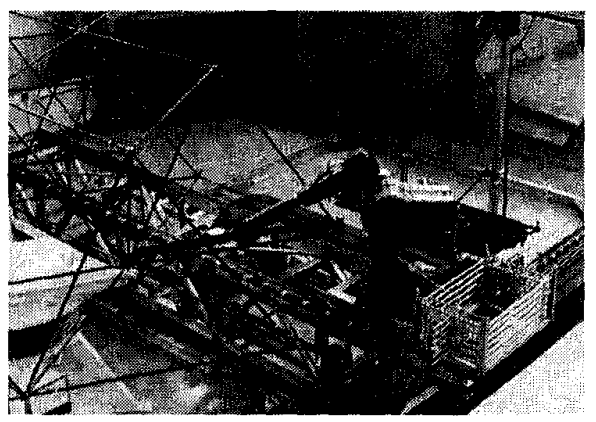

(b) - Collision avoidance during EVR truss assembly

Figure 13 - Key driver of both EVA and EVR truss assembly rate is the ease of operation of hardware

These projections indicate that two astronauts could assemble a $25 \mathrm{~m}$ reflector in roughly 12 hours, or the time associated with two EVA s. Even if the actual time required on orbit is twice as long as the time predicted from these tests (i.e., 24 hours versus 12 hours), the total EVA time is still within the capability of a single Shuttle mission.

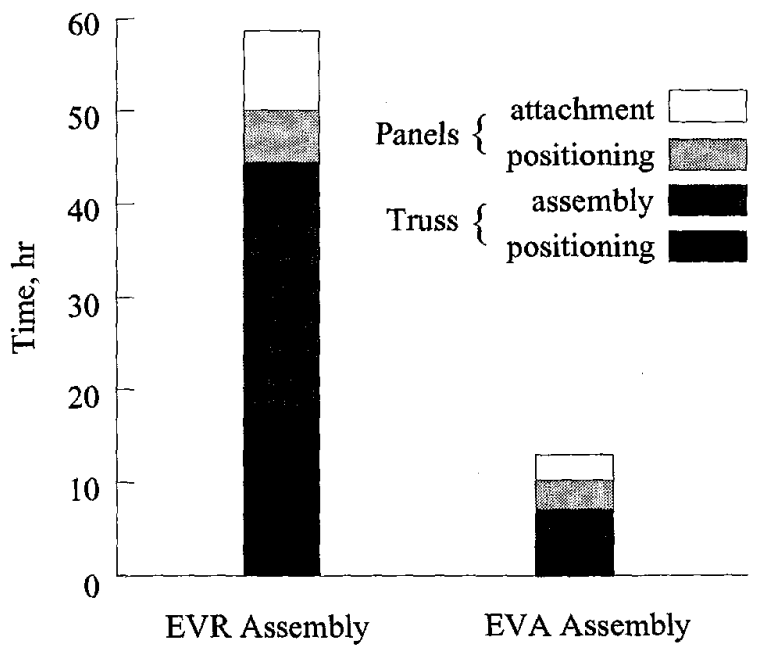

Figure 14 - Predictions of the time to build a 25m, 36-panel reflector on orbit 
It should be noted here that neither of the EVA and EVR assembly tests discussed in [10] and [11] included installation of wiring and utilities associated with active control systems for the mirror segments. Addition of such equipment would certainly increase the assembly times beyond those estimated in Figure 14.

\section{STRUCTURAL REQUIREMENTS (WHAT IS REQUIRED TO APPLY NGST ACTIVE CONTROL TECHNOLOGY TO A 25M TELESCOPE?)}

With the issues of launch vehicle packaging and on-orbit assembly having been addressed, it is now important to consider the issue of on-orbit structural performance of the mirror. For the present study, the main driver of structural requirements is a system-level requirement to accommodate the mirror panel and active alignment-control technology currently under development for the NGST. In this section, it will be shown that applying NGST mirror and active control technology to a $25 \mathrm{~m}$ telescope requires the fundamental frequency of the $25 \mathrm{~m}$ telescope to be approximately the same as that of the $8 \mathrm{~m}$ NGST. Relaxation of this frequency requirement implies that the $25 \mathrm{~m}$ mirror might need a more robust active-control system than the one to be developed for NGST.

\section{Quasi-Static Loads and Passive Stiffness Requirements}

The disturbance loads that can affect the dimensional precision of a large space telescope can include slew loads, gravity gradient loads, solar- and atmospheric-drag loads, and loads from on-board machinery (e.g., CMG and cryocooler). Except for the disturbances due to on-board machinery, most other disturbances are slowly varying in time, and optical distortions due to these loads can be estimated by static-response analysis [13]. An upper bound on the root-mean-square (rms) magnitude of deformation in a telescope mirror due to static loading is given by:

$$
\mathrm{x}_{\mathrm{rms}} \leq \frac{\mathrm{a}_{\mathrm{rms}}}{4 \pi^{2} \mathrm{f}_{0}^{2}}
$$

where $f_{0}$ is the fundamental natural frequency of the body (in $\mathrm{Hz}$ ), and $\mathrm{a}_{\mathrm{rms}}$ is the rms magnitude of the applied inertial acceleration. It should be noted that Equation (4) does not include any structural dimensions. This result is the reason that two different sized telescopes with equal vibration frequencies can be expected to have similar response magnitudes for the same inertial disturbances.

Figure 15 presents a plot of Equation (4) for four values of the fundamental natural frequency, $\mathrm{f}_{0}$ (ranging from $0.1 \mathrm{~Hz}$ to $100 \mathrm{~Hz}$ ). The abscissa represents the inertial disturbance magnitude, $a_{\text {rms, (in }}$ g s) and the ordinate represents the elastic deformation magnitude, $x_{\text {tms }}$. Overlaid within Figure 15 are approximate disturbance-rejection bands for both ground and space telescopes. These bands indicate the rough-order-of-magnitude upper and lower bounds on disturbance accelerations and response magnitudes that must be accommodated for by the telescope structure and/or active alignment control system.

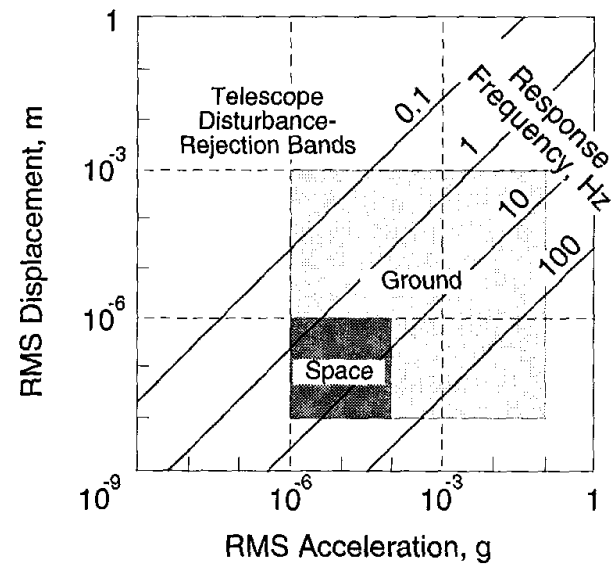

Figure 15 - Relationship between displacement, acceleration, and response frequency for static loading

Figure 15 provides a tool for quick assessment of the trade between passive stiffness of a telescope structure and active alignment control systems. Given a value of structural response frequency, the line associated with that frequency provides an upper bound on the elastic response of the structure across the spectrum of static disturbance loads. Therefore, the portion of the disturbance-rejection band above the response frequency line is accommodated passively by the inherent stiffness of the structure. On the other hand, the portion of the disturbance-rejection band below the response frequency line must be accommodated by the active control (or isolation) system.

To further illustrate this point, ground-based telescope systems are traditionally designed to exhibit vibration frequencies on the order of hundreds of $\mathrm{Hz}$. Figure 15 indicates that most of the ground-based disturbancerejection band is accommodated passively by telescope systems with frequencies above about $200-300 \mathrm{~Hz}$ (i.e., most of the ground telescope disturbance-rejection band is above the $200-300 \mathrm{~Hz}$ line). Similarly, the HST exhibits fundamental frequencies well above $50 \mathrm{~Hz}$, and accommodates essentially all on-orbit disturbances passively (i.e., most of the space telescope disturbancerejection band is above the $50 \mathrm{~Hz}$ line).

The optical telescope assembly (OTA) of the NGST is expected to exhibit a fundamental frequency of about $10 \mathrm{~Hz}$. Figure 15 indicates that this level of stiffness will enable the NGST to reject a large portion of the possible disturbance loads passively. In order to ensure stability over the entire band of possible disturbances, the NGST OTA will probably be passively and/or actively isolated from the NGST spacecraft. Finally, the NGST will incorporate quasi-static re-alignment capability in its primary mirror to accommodate creep and other long-term material instabilities. 


\section{Effects of Dynamic Loads and Damping}

As mentioned earlier, the main sources of dynamic loads for a space telescope are on-board machinery such as cryocoolers and reaction-control wheels. Typically, highfrequency components of the disturbance spectrum from these devices will be attenuated to acceptable levels through active or passive isolation systems. Nevertheless, it is prudent to design for some level of broadband loading on the telescope assembly.

Next to fundamental frequency, the most important parameter for determining the response of a telescope structure to low-level, broadband loading is damping. The upper bound on response to static loading given in Equation (4), can be modified to give an upper bound on the response to broadband dynamic loading by including a magnification factor which accounts for system damping [13]. The resulting equation is:

$$
\mathrm{x}_{\mathrm{rms}} \leq\left(\frac{1}{2 \zeta}\right) \frac{\mathrm{a}_{\mathrm{rms}}}{4 \pi^{2} \mathrm{f}_{0}^{2}}
$$

where $\zeta$ is the modal damping coefficient. A typical value for damping in mechanically jointed structures is $\zeta^{-} 0.01$, which leads to a magnification factor $(1 / 2 \zeta)$ of 50 ! In other words, persistent cyclic disturbances with broadband frequency content (encompassing the fundamental modal frequency of the structure) can easily cause elastic deformations several orders of magnitude larger than those that would result from static loading.

To make matters worse, many future space telescopes (including NGST) are being designed to operate at cryogenic temperatures where passive damping coefficients are expected to be substantially lower than 0.01 . This result clearly demonstrates the importance of attenuation of broadband disturbances through isolation of the telescope assembly or addition of passive or active damping to the telescope structure. Both of these options are being explored for the NGST, and would probably be necessary for $25 \mathrm{~m}$ space telescope. In general however, the best mitigation strategy for the risk associated with uncertainty in damping and persistent broadband disturbances is to design the fundamental frequency of the telescope assembly as high as possible.

\section{Achieving a $10 \mathrm{~Hz}$ Vibration Frequency in a $25 \mathrm{~m}$ Mirror}

The minimum frequency goal for the NGST optical telescope assembly is $10 \mathrm{~Hz}$. How does this goal translate to a $25 \mathrm{~m}$ diameter, NGST-derivative telescope? Simply put, as long as the larger telescope can be designed to exhibit the same minimum frequency (i.e., $10 \mathrm{~Hz}$ ), it is reasonable to assume that the active control problem on the $25 \mathrm{~m}$ system is no more difficult than that for the $8 \mathrm{~m}$ NGST.

At this point, it is important to determine the feasibility of achieving a $10 \mathrm{~Hz}$ first frequency in a $25 \mathrm{~m}$ mirror.
Specifically, it is important to quantify the structural mass necessary to achieve a $10 \mathrm{~Hz}$ fundamental frequency in a $25 \mathrm{~m}$ mirror in order to assess the feasibility of achieving an overall deployed areal density of $15 \mathrm{Kg} / \mathrm{m}^{2}$.

In [14] a detailed analysis is presented for the free-free vibration of tetrahedral truss platforms supporting segmented reflectors (like the erectable reflector concept presented herein). The analyses in [14] consider segmented reflectors of various diameters and number of panels. The analyses are based on a model of the reflector in which the reflector panels, mechanical joints, and any other nonstructural components are treated as parasitic mass and the stiffness is determined by an equivalent sandwich plate representation of the truss (see Figure 16).

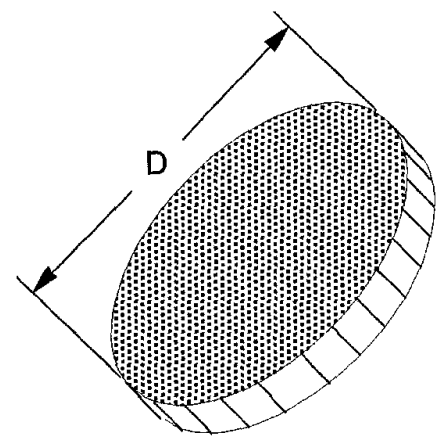

Equivalent Sandwich Plate

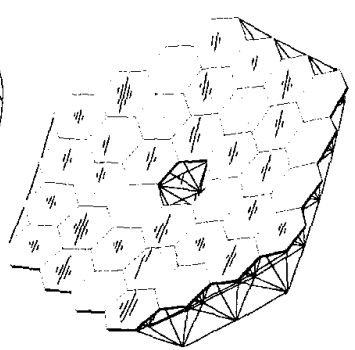

Reflector System
Figure 16 - Equivalent sandwich-plate model of trusssupported, segmented reflector from [9]

In the appendix, it is shown that the fundamental, free-free vibration frequency for a 36-panel, truss-supported, erectable reflector (Figures 7 and 16) reduces to:

$$
\left(f_{0}\right)_{36-p a n e l}=\frac{0.58}{2 \pi D} \sqrt{\eta(E / \rho)_{\text {truss }}}
$$

where $D$ is the diameter of the reflector, $(E / \rho)_{\text {truss }}$ is the specific stiffness of the material in the support truss, and $\eta$ is the fraction of the total reflector mass allotted to the support truss and defined (in the Appendix) by:

$$
\eta=\frac{\left(\rho_{\text {areat }}\right)_{\text {structural }}}{\left(\rho_{\text {areal }}\right)_{\text {parasitic }}+\left(\rho_{\text {areat }}\right)_{\text {structural }}}
$$

Note that these analyses assume that the lowest vibration frequency of the mirror is the frequency of its fundamental free-free vibration mode. However, a mirror attached at its center to a spacecraft or science instrument module is likely to exhibit a fundamental vibration frequency lower than the value predicted for the free-free case.

Figure 17 presents a plot of the value of vibration frequency (determined from Equation (6)) as a function of reflector diameter for various values of $\eta$. In this plot it is assumed 
that the reflector support truss is made of a graphite/epoxy material with $E=30 \times 10^{6}$ psi and $\rho=0.06 \mathrm{lb} / \mathrm{in}^{3}$.

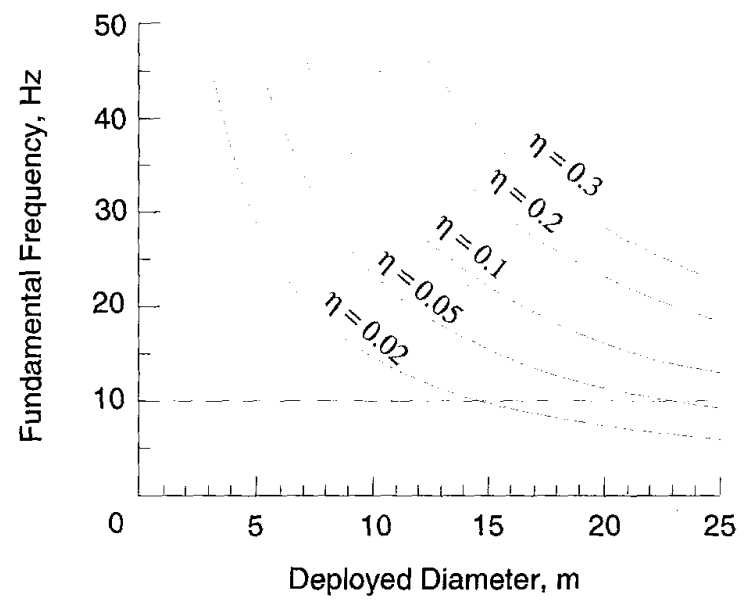

Figure 17 - Approximate fundamental vibration frequency of 36-panel, erectable reflector

From Figure 17, it can be seen that a $25 \mathrm{~m}$ erectable reflector can easily be designed to exhibit a $10 \mathrm{~Hz}$ first frequency with a very modest fraction (i.e., $\eta=0.06$ or $6 \%$ ) of the total mass invested in a deep truss using a reasonably efficient structural material (i.e., graphite/epoxy). It is important to restate here that this structural mass fraction is only the material making up the truss struts, it does not include the parasitic mass associated with the truss such as the mechanical joints between the members. To account for non-structural mass in the truss and allow for the fact that the actual fundamental frequency should be below the freefree vibration frequency, the structural mass fraction computed can be inflated by a factor of four. Multiplying the computed mass fraction of $6 \%$ by four gives an estimate of $25 \%$ for the fraction of the reflector mass that must be invested in the truss structure to ensure a first frequency of $10 \mathrm{~Hz}$.

In summary, these analyses indicate that it is reasonable to expect that a $25 \mathrm{~m}$ mirror could be designed that exhibits an overall deployed areal density of $15 \mathrm{Kg} / \mathrm{m}^{2}$ and a first frequency of $10 \mathrm{~Hz}$. However, achieving this design REQUIRES the use of an erectable mirror architecture with a deep truss structure (i.e., roughly $3 \mathrm{~m}$ deep for a $25 \mathrm{~m}$ diameter reflector) constructed of a moderately efficient material such as a graphite-fiber-reinforced composite.

\section{SUMMARY}

This study was motivated by the observation that, as a consequence of an order of magnitude reduction in areal density, the NGST primary mirror will be volume critical in its launch package whereas the HST mirror was mass critical. This result implies that mirrors substantially larger than the $8 \mathrm{~m}$ NGST mirror, but with the same areal density as the NGST mirror, can be feasible if efficient schemes for packaging the mirrors in a launch vehicle can be devised.

This study focused on the design of a $25 \mathrm{~m}$-diameter telescope mirror simply because this size gives a tenfold increase in collecting area from the NGST. Of course, $20 \mathrm{~m}$ class space telescopes are currently on the wish list of the visible and infrared astronomy community for the 2010 to 2020 timeframe, so focusing on a $25 \mathrm{~m}$ aperture size implicitly addresses the future needs for space-based observatories.

This study was bounded by the practical goal of applying both mirror panel and active-alignment-control technologies currently under development for the NGST to a $25 \mathrm{~m}$ telescope mirror. Consequently, the present study did not consider extremely lightweight (i.e., areal densities less than $1 \mathrm{~kg} / \mathrm{m}^{2}$ ) gossamer telescopes using thin membrane optics coupled with active wave-front control (e.g., Figure 2). Clearly, if proven viable, membrane optics might dramatically reduce launch costs for large space telescopes, and should be seriously considered for application to $25 \mathrm{~m}$ observatories in the future.

With these general assumptions, the present study has considered three key issues for the design of a $25 \mathrm{~m}$, space telescope mirror: launch vehicle packaging; on-orbit assembly; and on-orbit structural performance. The general conclusions of the study are as follows:

- In order to achieve a volumetric packaging efficiency consistent with a deployed areal density of $15 \mathrm{~kg} / \mathrm{m}^{2}$, a $25 \mathrm{~m}$ mirror must be assembled on-orbit instead of mechanically deployed.

- The on-orbit assembly of a $25 \mathrm{~m}$ mirror is within the capability of either astronauts in EVA or extravehicular robotic (EVR) systems. The selection between EVA and EVR assembly should be based on practical and economic considerations such as the availability of both EVA and EVR systems.

- Achieving a fundamental frequency of $10 \mathrm{~Hz}$ in an erectable, truss-supported, $25 \mathrm{~m}$ reflector (and thus ensuring applicability of NGST mirror panel and active control technology) requires no more than $25 \%$ of the total mass of the reflector system to be invested in the underlying truss. This modest requirement for structural mass fraction indicates that it is reasonable to expect that a $25 \mathrm{~m}$ mirror could be designed that exhibits overall deployed areal density of $15 \mathrm{Kg} / \mathrm{m}^{2}$.

- Launching a space telescope observatory with a $25 \mathrm{~m}$ segmented primary mirror would require the dedicated resources of a single Shuttle (or Titan IV) mission assuming that on-orbit assembly and initial check-out is performed in low Earth orbit. 


\section{REFERENCES}

[1] Dressler, Alan, ed.: Exploration and the Search for Origins: A Vision for Ultraviolet-Optical-Infrared Astronomy, Report of the HST and Beyond Committee, Association of Universities for Research in Astronomy, May 1996.

[2] Bely, Pierre, Y., Perrygo, Charles, and Burg, Richard., NGST Yardstick Mission, NGST Monograph No. 1, Next Generation Space Telescope Project Office, NASA Goddard Space Flight Center, July 1999.

[3] Mahoney, M. J.; and Ibbott, A. C.; and Cassingham, Randy: A Large Deployable Reflector Assembly Scenario A Space Station Utilization Study, JPL Document D-5942, November 1988.

[4] Thompson, Mark W.: The AstroMESH Deployable Reflector, Proceedings of the $5^{\text {th }}$ International Satellite Conference, (IMSC 97), Pasadena, CA, June 16-17, 1997, JPL Publication 97-11, 1997, pp. 393-398.

[5] Chmielewski, A. B.; Noca, Muriel; and Wietfeldt, R. D.: ARISE Advanced Radio Interferometry between Space and Earth, JPL Publication 99-14, October, 1999.

[6] Isakowitz, Steven J.: International Reference Guide to Space Launch Systems, American Institute of Aeronautics and Astronautics, Inc, '1991.

[7] Mikulas, Martin M.; Lou, Michael C.; Withnell, Peter R.; and Thorwald, Gregory: Deployable Concepts for Precision Segmented Reflectors, JPL D-10947, June, 1993.

[8] Mikulas, Martin M. Jr.; Collins, Timothy J.; and Hedgepeth, John M.: Preliminary Design Approach for Large High Precision Segmented Reflectors, NASA TM102605, February, 1990.

[9] Mikulas, Martin M. Jr.; Collins, Timothy J.; and Hedgepeth, John M.: Preliminary Design Considerations for 10 to 40 Meter-Diameter Precision Truss Reflectors, Journal of Spacecraft and Rockets, Fol. 28 No. 4, 1991.
[10] Lake, Mark S.; Heard, Walter L.; Watson, Judith J.; and Collins, Timothy J.: Evaluation of Hardware and Procedures for Astronaut Assembly and Repair of Large Precision Reflectors, NASA TP-2000-210317, August, 2000.

[11] Rhodes, Marvin D.; Will, Ralph W.; and Quach, Coung: Baseline Tests of an Autonomous Telerobotic System for Assembly of Space Truss Structures, NASA TP3448, July, 1994.

[13] Lake, Mark S.; Peterson, Lee D.; and Levine, Marie B.: A Rationale for Defining Structural Requirements for Large Space Telescopes, to be presented at the $42^{\text {nd }}$ AIAA, ASME/AHS Structures, Structural Dynamics, and Materials Conference, April 16-19, 2001, Seattle, WA.

[14] Wu, K. Chauncey; and Lake, Mark S.: Natural Frequency of Uniform and Optimized Tetrahedral Truss Platforms, NASA TP-3461, November, 1994.

Mark Lake received a B.S. in Aeronautical and Astronautical Engineering from the University of Illinois in 1984, an M.S. in Mechanical Engineering from old Dominion University in 1989, and a Ph.D. in Aerospace Engineering from North Carolina State University in 1992. He has been employed by NASA Langley

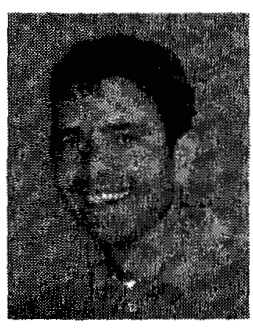
Research Center since 1981. Since 1984, he has performed research in theoretical structural mechanics, and has designed, developed, and tested numerous advanced hardware concepts for spacecraft and launch vehicle structures. Since 1994, he has conducted research on highprecision deployable structures specifically for application to space telescopes and interferometers. Currently, Dr. Lake is on temporary assignment to the Jet Propulsion Laboratory. 


\section{APPENDIX \\ (SOLUTION FOR FUNDAMENTAL VIBRATION FREQUENCY OF A TRUSS-SUPPORTED REFLECTOR)}

The analyses presented in both [9] and [14] for the fundamental vibration frequency of a tetrahedral-trusssupported, segmented reflector are based on an equivalent, circular, sandwich-plate representation of the reflector (as sketched in Figure 16). The general solution for the fundamental free-free vibration frequency derived in [14] is:

$$
f_{0} \approx \frac{\kappa}{2 \pi D} \sqrt{\eta(E / \rho)_{\text {Iruss }}}
$$

where $\kappa$ is an empirical constant that depends on the number of rings in the reflector [see Figure (A1)], $D$ is the maximum reflector diameter, $(E / \rho)_{\text {truss }}$ is the specific modulus of the truss material, and $\eta$ is the structural mass fraction defined by:

$$
\eta=\frac{\left(\rho_{\text {areal }}\right)_{\text {structurat }}}{\left(\rho_{\text {areal }}\right)_{\text {parasitic }}+\left(\rho_{\text {areal }}\right)_{\text {structural }}}
$$

In Equation (A2), $\left(\rho_{\text {areal }}\right\rangle_{\text {structural }}$ is the structural areal density, defined by the total mass of the truss (minus parasitic mass components such as joints) divided by the total area of the reflector. Similarly, the parasitic areal density, ( $\left.\rho_{\text {areal }}\right)_{\text {parasitic, }}$ is the total parasitic mass divided by the total area of the reflector.

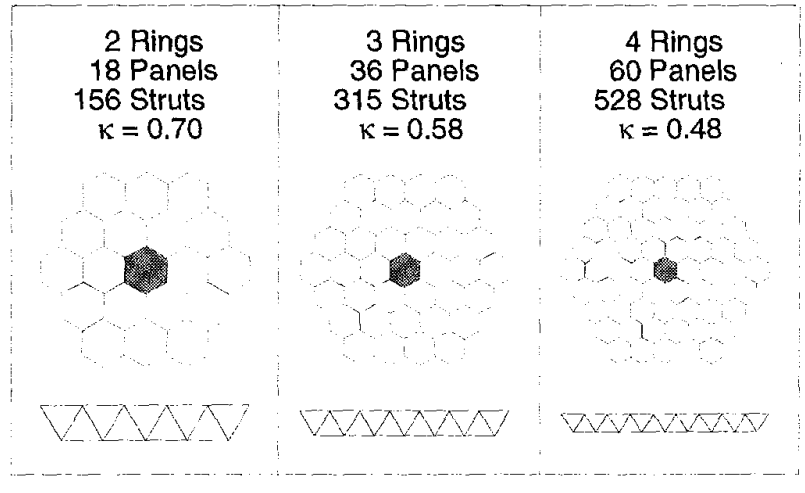

Figure A1 - Part counts and empirical frequency constant for segmented reflectors with 2 to 4 rings of panels.

The reflector considered in the present study (and shown in Figure 7) includes 36 panels arranged in three concentric rings. Substituting $\kappa=0.58$ into Equation (A1) gives the following equation for the frequency of a 36-panel reflector:

$$
\left(f_{0}\right)_{36-p a n e l}=\frac{0.58}{2 \pi D} \sqrt{\eta(E / \rho)_{\text {thuss }}}
$$

One final note: these analyses assume that the lowest vibration frequency of the mirror is the frequency of its fundamental free-free vibration mode. However, a mirror attached at its center to a spacecraft or science instrument module is likely to exhibit a fundamental vibration frequency lower than the value predicted for the free-free case. 